Pacific Journal of Mathematic 


\section{TWO REMARKS ON POLYNOMIALS IN TWO VARIABLES}

\section{Shulim Kaliman}

Let $X$ be a compactification of $\mathbf{C}^{2}$ such that a polynomial $p$ can be extended to a regular mapping $\bar{p}: X \rightarrow \mathbf{C P}^{1}$. If generic fibers of $p$ are irreducible, then we show that the number of reducible fibers is less than the number of horizontal components of the curve $X-\mathrm{C}^{2}$. If $p$ is rational, then the restriction of $\bar{p}$ to every horizontal component except one is a one-to-one mapping.

Introduction. Let $p \in \mathbf{C}[x, y]$ be a polynomial in two complex variables. Recall that a polynomial fiber is a set

$$
\Gamma_{c}=\left\{(x, y) \in \mathbf{C}^{2} \mid p(x, y)=c\right\},
$$

where $c \in \mathbf{C}$, i.e., every polynomial fiber is an affine algebraic curve. There is a finite set $S \subset \mathbf{C}$ such that for every $c, c^{\prime} \in \mathbf{C}-S$ the fibers $\Gamma_{c}$ and $\Gamma_{c^{\prime}}$ are homeomorphic. If $c \in \mathbf{C}-S$, then the fiber is called generic (the definition of the generic fibers of a polynomial is a little different, but we can use this one according to [LZ]). A polynomial is called primitive if its generic fibers are connected. For every non-primitive polynomial $q(x, y)$ there exist a primitive polynomial $p(x, y)$ and a polynomial $h(z)$ in one variable such that $q(x, y)=h(p(x, y))[\mathbf{L Z}],[\mathbf{F}]$. This fact reduces the study of polynomials in two variables to the case of primitive polynomials. From now on we shall restrict ourselves to primitive polynomials only. If a fiber of $p$ is not homeomorphic to a generic fiber, it is called a degenerate fiber. A degenerate fiber can be reducible even when $p$ is primitive, in other words this fiber can consist of more than one irreducible component. Let $k$ be the degree of a primitive polynomial $p$. Then the number $r$ of reducible fibers is less than $k-1$ [St]. V. Ya. Lin knew, but never published a theorem from which a stronger statement follows: if the polynomial $p$ has type $(g, m)$ (i.e., its generic fibers are $m$-punctured Riemann surfaces of genus $g$ ), then the number of reducible fibers is less than $m-1$, and it is easy to check that $m \leq k$. Our first aim is to improve these estimates.

Standard results of the theory of resolution of singularities guarantee the existence of a smooth compactification $\bar{X}$ of $\mathbf{C}^{2}$ such that the 
mapping $p: \mathbf{C}^{2} \rightarrow \mathbf{C}$ can be extended to a regular mapping $\bar{p}: \bar{X} \rightarrow$ $\mathbf{C P}^{1}$. Then $\mathscr{D}=\bar{X}-\mathbf{C}^{2}$ is an algebraic curve, every component of which is isomorphic to $\mathbf{C P}^{1}$. A component $E$ of $\mathscr{D}$ is called horizontal if the restriction of $\bar{p}$ to $E$ is not a constant mapping. Of course, the number $n$ of horizontal components is less then $m$. We shall prove that $r \leq n-1$. In the case when $p$ is a rational polynomial (i.e., its generic fibers are $m$-punctured Riemann spheres) we have a more exact estimate (this case was considered also in [Sa]). These results are contained in the first section. A component $E$ of the curve $\mathscr{D}=\bar{X}-\mathrm{C}^{2}$ is called a section if the restriction of $\bar{p}$ to $E$ is a one-to-one mapping.

In some cases every horizontal component of $\mathscr{D}$ is a section. It is so, for instance, when the leading terms of the polynomial $p$ form a homogeneous polynomial without multiple roots. Another example is the Abhyankar-Singh theorem [AS]. This theorem says that if a polynomial fiber is a once-punctured Riemann surface and, therefore, there is only one horizontal component, then this component is a section. This fact plays a very important role in such classification theorems about smooth polynomial embeddings of once-punctured Riemann surfaces into $\mathbf{C}^{2}$ as the Abhyankar-Moh-Suzuki theorem [AM] and Neumann's theorem $[\mathbf{N}]$.

In the general case there exist polynomials with horizontal components different from sections. Nevertheless for rational polynomials there is a weaker analog of the Abhyankar-Singh theorem. The result of the second section is the following assertion (Theorem 5), which first was conjectured by Bogomolov.

If $p$ is a rational polynomial, then at most one horizontal component of the curve $\mathscr{D}=\bar{X}-\mathbf{C}^{2}$ is not a section.

Theorem 5 enables us to use the technique of $[\mathbf{Z 2}]$ and [S] in the study of rational polynomials. In particular we have obtained the classification of rational polynomials with a $\mathbf{C}^{*}$-fiber $[\mathbf{K}]$ due to this approach.

1. Reducible fibers. Let $\bar{p}: \bar{X} \rightarrow \mathbf{C P}$ be a regular extension of a primitive polynomial $p: \mathbf{C}^{2} \rightarrow \mathbf{C}$ and $\mathscr{D}=\bar{X}-\mathbf{C}^{2}$. Using the blowup process, if necessary, one may suppose that $\bar{X}$ is smooth (note that this blow-up process does not change the number of horizontal components of $\mathscr{D})$. Let $F_{c}=\bar{p}^{-1}(c)$ for $c \in \mathbf{C P}^{1}$. Suppose that $S \subset \mathbf{C}$ is the set of degenerations of $p$, i.e., a fiber $\Gamma_{c}=\{(x, y) \in$ $\left.\mathbf{C}^{2} \mid p(x, y)=c\right\}$ is degenerate iff $c \in S$. Then one may suppose [ $\mathbf{Z 1}$, Proposition 3.6] that $F_{c}$ is a degenerate fiber of $\bar{p}$, iff $c \in \bar{S} \stackrel{\text { def }}{=} S \cup \infty$. 
THEOREM 1. Let $F$ be a generic fiber of $\bar{p}, g$ be the genus of $F, k_{s}=\operatorname{dim} H_{1}(F)-\operatorname{dim} H_{1}\left(F_{s}\right)$ for $s \in \bar{S}, r_{s}$ be the number of irreducible components of components of the fiber $\Gamma_{s}$ (in other words $\Gamma_{s}$ consists of $r_{s}$ different algebraic curves), and $n$ be the number of horizontal components of $\mathscr{D}$. Then

$$
\sum_{s \in S} k_{s}=2 g+n-1-\sum_{s \in S}\left(r_{s}-1\right) .
$$

Proof. Let $m$ be the number of components of $\mathscr{D}$. Note that the Euler characteristics $\chi(\bar{X})$ of $\bar{X}$ is $2+m$. We shall denote the Euler characteristics of $F$ and $F_{S}$ by $\chi(F)$ and $\chi\left(F_{S}\right)$ respectively. Then the following formula holds [Sh]:

$$
\sum_{s \in \bar{S}} \chi\left(F_{S}\right)-\chi(F)=\chi(\bar{X})-2 \chi(F) .
$$

Let $m_{\infty}$ be the number of irreducible components of $F_{\infty}=\bar{p}^{-1}(\infty)$ (each of them is isomorphic to $\mathbf{C P}^{1}$ ), and let $m_{s}$ be the number of irreducible components of $F_{s}$ such that these components belong to $\mathscr{D}$. Then $\chi\left(F_{\infty}\right)-\chi(F)=1+m_{\infty}-(2-2 g), m=\sum_{s \in \bar{S}} m_{s}+n$, and $r_{s}+m_{s}$ is the number of irreducible components of $F_{s}$. Now we can rewrite (1) in the following way

$$
\begin{aligned}
\sum_{s \in S} & \left(\chi\left(F_{s}\right)-\chi(F)\right)+1+m_{\infty}-(2-2 g) \\
= & 2+m_{\infty}+\sum_{s \in S} m_{s}+n-2(2-2 g) .
\end{aligned}
$$

Hence

$$
\sum_{s \in S}\left(\chi\left(F_{S}\right)-\chi(F)\right)=2 g-1+\sum_{s \in S} m_{s}+n .
$$

Since the fiber $F_{S}$ is connected, we have

$$
\chi\left(F_{s}\right)-\chi(F)=-1+k_{s}+r_{s}+m_{s} .
$$

Thus (2) implies

$$
\sum_{s \in S} k_{s}+\sum_{s \in S}\left(r_{s}-1\right)+\sum_{s \in S} m_{s}=2 g-1+n+\sum_{s \in S} m_{s} .
$$

And finally we have

$$
\sum_{s \in S} k_{s}=2 g-1+n-\sum_{s \in S}\left(r_{s}-1\right) .
$$


We would like to emphasize that the formulation and the proof of this theorem does not depend on whether or not the irreducible components of the degenerate fibers are multiple.

CoRollaRY 2. Under the assumptions of Theorem 1 the following inequality holds $n-1 \geq \sum_{s \in S}\left(r_{s}-1\right)$. If $p$ is a rational polynomial, then $n-1=\sum_{s \in S}\left(r_{s}-1\right)$.

Proof. Since $\left(\bar{X}, F_{S}\right)$ is a polyhedral pair [L], $F_{S}$ is a strong deformation retract of its sufficiently small neighborhood $U$ [Sp]. Choose small closed discs $\Delta_{s}^{\prime} \subset \Delta_{s}$ around $s \in S$ so that for $K_{s}=\bar{p}^{-1}\left(\Delta_{S}\right)$ and $K_{s}^{\prime}=\bar{p}^{-1}\left(\Delta_{s}^{\prime}\right)$ we have $K_{s} \supset U \supset K_{s}^{\prime}$. One may suppose that $\Delta_{s} \cap S=s$. Then $K_{s^{\prime}}$ is a strong deformation retract of $K_{s}$. Hence one can easily check that $F_{S}$ is a strong deformation retract of $K_{s}$ as well. The imbedding of a generic fiber $F \hookrightarrow K_{S}$ induces the mapping $\rho_{s}: H_{1}(F) \rightarrow H_{1}\left(K_{s}\right)=H_{1}\left(F_{s}\right)$. Clearly, $H_{1}\left(F_{s}\right)$ contains no element of finite order. Thus $k_{s}=\operatorname{dim} \operatorname{ker} \rho_{s}=\operatorname{dim} H_{1}(F)-\operatorname{dim} H_{1}\left(F_{s}\right)$. Let $X^{*}=\bar{X}-F_{\infty}$. Then $\bar{p}\left(X^{*}\right)=\mathbf{C}$ and $H_{1}\left(X^{*}\right)=0$, since $\mathbf{C}^{2}$ is dense in $X^{*}$. Let $V$ be a disjoint union of sets $\left\{K_{s}=\bar{p}^{-1}\left(\Delta_{s}\right) \mid s \in S\right\}$ so that $F_{S}$ is a strong deformation retract of $K_{s}$ for each $s \in S$. Let $A$ be a closed connected simply connected subset of $\mathbf{C}$ such that the set $A \cap \Delta_{s}$ consists of one point for each $s \in S$. Then $A \cup \bigcup_{s \in S} \Delta_{S}$ is a strong deformation retract of C. Put $W=\bar{p}^{-1}(A)$. Obviously $W \cup V$ is a strong deformation retract of $X^{*}$, and $H_{1}(V \cap W)=\bigoplus_{s \in S} H_{1}(F)$, $H_{1}(W)=H_{1}(F), H_{1}(V)=\bigoplus_{s \in S} \rho_{s}\left(H_{1}(F)\right)$. It follows from the Mayer-Vietoris sequences

$$
\bigoplus_{s \in S} H_{1}(F) \stackrel{j}{\longrightarrow} H_{1}(F) \bigoplus_{s \in S} \rho_{s}\left(H_{1}(F)\right) \rightarrow 0 .
$$

Thus $j\left(\bigoplus_{s \in S} H_{1}(F)\right)$ contains the subgroup $G=H_{1}(F) \oplus 0 \oplus \cdots \oplus 0$. Clearly $j^{-1}(G) \subset \bigoplus_{s \in S} \operatorname{ker} \rho_{s}$. Since $j$ is surjective, this implies $\bigoplus_{s \in S} \operatorname{dim} \operatorname{ker} \rho_{s}=\sum_{s \in S} k_{s} \geq 2 g$. If $g=0$, then $\bigoplus_{s \in S} \operatorname{dim} \operatorname{ker} \rho_{s}=$ $2 g=0$. Now Theorem 1 implies the desired conclusion.

REMARK. Let $X$ be an acyclic surface (i.e., $H_{k}(X)=0$ whenever $k>0$ and $X$ is connected), and let $p: X \rightarrow \mathbf{C}$ be a regular mapping. Then again there exists a smooth compactification $\bar{X}$ of $X$ such that $p$ extends to a regular mapping. We can define in the same way primitive mappings, reducible fibers of $p$, the curve $\mathscr{D}=\bar{X}-X$, its horizontal components and sections. Then it is easy to see that the formulation and the proof of Theorem 1 and Corollary 2 can be carried over completely to this case as well. 
2. Rational polynomials. First we recall some terminology. If $G$ is a connected graph and $e$ is a vertex of $G$, then connected components of the graph obtained by removing $e$ from the vertices of $G$ and deleting the links at $e$ are called branches of $G$ at $e$. The vertex $e$ is a branch point, if the number of branches at $e$ is at least three, otherwise $e$ is a linear point. If a connected graph has no branch points we call it linear. Let $\mathscr{D}$ be a complete algebraic curve in a compact algebraic surface $\bar{X}$. As usual the weighted graph $G(\mathscr{D})$ corresponds to $\mathscr{D}$. Each vertex $G(\mathscr{D})$ corresponds to an irreducible component of $\mathscr{D}$; if an irreducible component meets another one, then the corresponding vertices are connected by the edge, and the weight of any vertex coincides with the intersection number of the corresponding component of $\mathscr{D}$. We say that the curve $\mathscr{D}$ has simple normal points of selfintersection, if every point of selfintersection of $\mathscr{D}$ belongs to two components of $\mathscr{D}$ only, and these components meet normally at this point. It is well known that every smooth compactification $\bar{X}$ of $\mathbf{C}^{2}$ is an algebraic surface and $\bar{X}$ can be chosen in such a way that the curve $\mathscr{D}=\bar{X}-\mathbf{C}^{2}$ has simple normal points of selfintersection. Every component of $\mathscr{D}=\bar{X}-\mathbf{C}^{2}$ is isomorphic to $\mathbf{C P} \mathbf{P}^{1}$. The Castelnuovo theorem $[\mathbf{G H}]$ implies that a component of $\mathscr{D}$ can be contracted if its selfintersection number is -1 . We shall need the following fact.

Theorem (Ramanujam-Morrow [R], [M]). Let $\bar{X}$ be an algebraic nonsingular complete surface. Let $\mathscr{D}$ be an algebraic curve in $\bar{X}$ with simple normal points of selfintersection. Suppose that $\bar{X}-\mathscr{D}$ is isomorphic to $\mathbf{C}^{2}$. Then successively contracting components corresponding to linear points of weight -1 one can reduce the curve $\mathscr{D}$ to a curve, whose weighted graph has one of the following representations where $l_{i} \leq-2, t_{j} \leq-2, n>0$.

2)

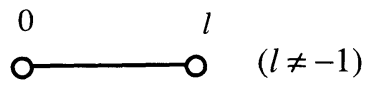

3)

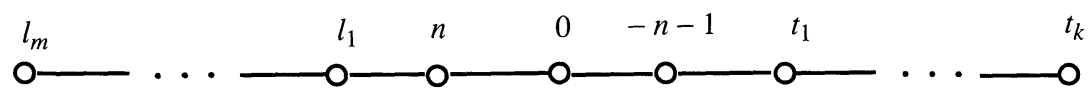

FIGURE 1 
COROllary 3. Let $\bar{X}$ be a smooth compactification of $\mathbf{C}^{2}$ such that $\mathscr{D}=\bar{X}-\mathbf{C}^{2}$ has simple normal points of selfintersection. Then the weighted graph $G(\mathscr{D})$ of $\mathscr{D}$ cannot have the following representation where each subgraph $G_{i}$ is nonempty and the weights of vertices $e$ and $f$ are -1 .

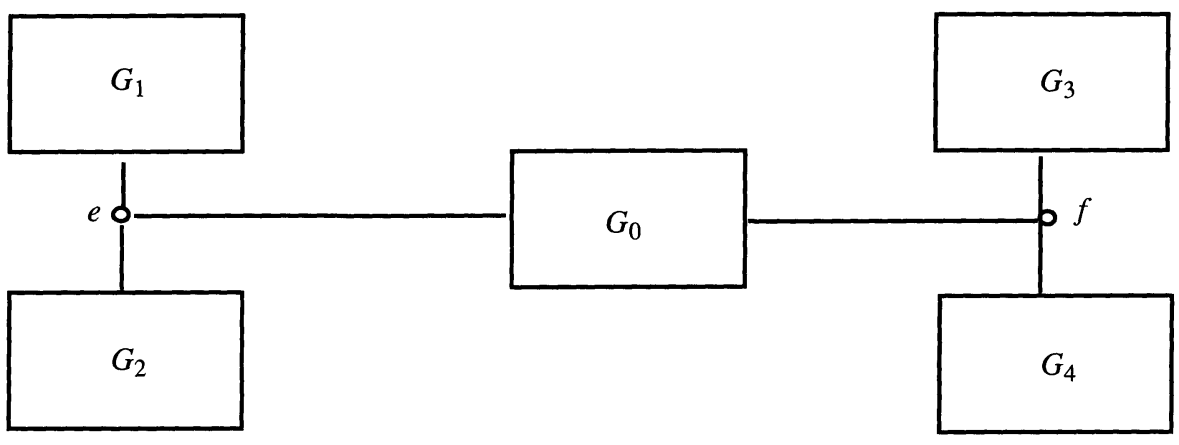

Figure 2

Proof. By the Ramanujam-Morrow theorem successively contracting components of $\mathscr{D}$ corresponding to linear points of weight -1 on each step we can obtain a linear graph. Assume that we contract all the components corresponding to the vertices of the subgraph $G_{0}$ before we can remove the branch points $e$ and $f$. Then the weights of these branch points become nonnegative, since we have to contract components with selfintersection -1 that meet the two curves corresponding to these two branch points. Thus we cannot contract these two curves further, for their selfintersection numbers can only increase during the next blow-up processes. Hence in order to obtain a linear graph we have to contract all components corresponding to the vertices of either the subgraph $G_{1}$ or the subgraph $G_{2}$, and all components corresponding to the vertices of either the subgraph $G_{3}$ or the subgraph $G_{4}$. Then the weights of the vertices $e$ and $f$ in the new graph become positive, and this contradicts the RamanujamMorrow theorem. Hence we cannot contract all the components of $\mathscr{D}$ that correspond to the vertices of the subgraph $G_{0}$, before we shrink one of the curves corresponding to $e$ or $f$. In order to obtain a linear graph we have to contract all components of $\mathscr{D}$ corresponding to the vertices of one of the subgraphs $G_{1}, G_{2}, G_{3}$, or $G_{4}$ (say $G_{1}$ ). Then the weight of $e$ in the new graph is nonnegative and the corresponding curve cannot be shrinked during the following blow-up processes. In the same way weight of $f$ becomes nonnegative, and finally we ob- 
tain two vertices with nonnegative weights, which are not neighboring, since the subgraph $G_{0}$ cannot be removed completely. This yields the desired contradiction with the Ramanujam-Morrow theorem.

LEMMA 4 (Zaidenberg). Let $p$ be a rational polynomial and $\bar{X}$ be a compactification of $\mathbf{C}^{2}$ such that the mapping $p: \mathbf{C}^{2} \rightarrow \mathbf{C}$ can be extended to a regular mapping $\bar{p}: \bar{X} \rightarrow \mathbf{C P}^{1}$. Let $H_{1}$ and $H_{2}$ be horizontal components of the curve $\mathscr{D}=\bar{X}-\mathrm{C}^{2}$. There is a commutative diagram

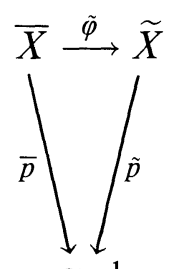

$\mathbf{C P}^{1}$

where

(1) $\tilde{X}$ is a compact surface and $\tilde{\varphi}$ is a birational isomorphism;

(2) if $\mathbf{C P}^{1}=\mathbf{C} \cup \infty, X^{*}=\bar{p}^{-1}(\mathbf{C})$, and $\widetilde{X}^{*}=\tilde{p}^{-1}(\mathbf{C})$, then $\left.\tilde{\varphi}\right|_{X^{*}}: X^{*} \rightarrow \widetilde{X}^{*}$ is a regular isomorphism;

(3) the fiber $\tilde{p}^{-1}(\infty)$ is irreducible;

(4) if $\widetilde{H}_{k}$ is the closure of $\tilde{\varphi}\left(H_{k} \cap X^{*}\right)$ in $\tilde{X}$, then $\widetilde{H}_{1} \cap \widetilde{H}_{2} \cap \tilde{p}^{-1}(\infty)$ $=\varnothing$.

Proof. Since the generic fiber of $\bar{p}: \bar{X} \rightarrow \mathbf{C P}^{1}$ is $\mathbf{C P}^{1}$, then $\bar{X}$ is a blow-up of a rational ruled surface $S_{n}$ and there is a commutative diagram [GH]

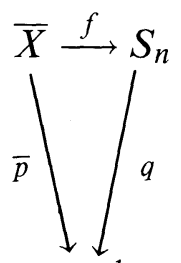

$\mathbf{C} \mathbf{P}^{1}$

In particular all components of the fiber $\bar{p}^{-1}(\infty)$ except one can be contracted, and we obtain a new surface $X_{1}$ and the commutative 
diagram

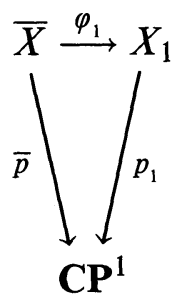

with irreducible fiber $E_{1}=p_{1}^{-1}(\infty)$. Moreover $\varphi_{1}$ is a regular morphism and the restriction of $\varphi_{1}$ to $X^{*}$ is a regular isomorphism between $X^{*}$ and $p_{1}^{-1}(\mathbf{C})$. Put $H_{k}^{1}=\varphi_{1}\left(H_{k}\right)$. If $H_{1}^{1} \cap H_{2}^{1} \cap E_{1}=\varnothing$ then the lemma is proved. Assume that $H_{1}^{1} \cap H_{2}^{1} \cap E_{1} \neq \varnothing$. Note that the Ramanujam-Morrow theorem easily implies that the curve $\mathscr{D}$ is simply connected. Thus $H_{k}^{1} \cap E_{1}$ consists of one point (say $a$ ). Blow up the surface $X_{1}$ at the point $a$ and then blow down the proper transform of $E_{1}$ (it is possible, since the selfintersection number of the fiber $E_{1}$ is 0 , and thus the selfintersection number of the proper transform of $E_{1}$ is -1 ). After this procedure we obtain a new surface $X_{2}$ such that there is a commutative diagram

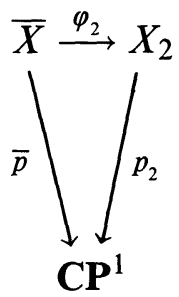

satisfying

(1) $\varphi_{2}$ is a birational isomorphism;

(2) $\left.\varphi_{2}\right|_{X^{*}}: X^{*} \rightarrow p_{2}^{-1}(\mathbf{C})$ is a regular isomorphism;

(3) $E_{2}=p_{2}^{-1}(\infty)$ is irreducible.

Let $H_{k}^{2}$ be the closure of $\varphi_{2}\left(H_{k} \cap X^{*}\right)$ in $X_{2}$. If $H_{1}^{2} \cap H_{2}^{2} \cap E_{2}=\varnothing$, then the lemma is proved. Otherwise it is easy to see that the contact order of the curves $H_{1}^{2}$ and $H_{2}^{2}$ at the point $H_{1}^{2} \cap H_{2}^{2} \cap E_{2}$ is less than the contact order of the curves $H_{1}^{1}$ and $H_{2}^{1}$ at the point $a$. Repeating the above procedure, one can reduce this contact order and finally obtain the desired commutative diagram.

Let $\widetilde{H}_{1}, \widetilde{H}_{2}, \widetilde{X}$ be as in Lemma 4. Standard results of the theory of resolution of singularities enable us to find such a blow-up $h: \widehat{X} \rightarrow \widetilde{X}$ 
that the curve $\widehat{\mathscr{D}}=h^{-1}(\widetilde{\mathscr{D}})$ has simple normal points of selfintersection. Moreover we can do it in such a way that the curve $\widehat{\mathscr{D}}$ is minimal in the following meaning: if we shrink a contractable component of $\widehat{\mathscr{D}}$, then the new curve contains selfintersection points that are not simple normal.

LEMMA 5. Let $\widetilde{H}_{1}$ and $\widetilde{H}_{2}$ not be sections. Then the weighted graph $G(\widehat{\mathscr{D}})$ of the curve $\widehat{\mathscr{D}}$ looks like in Corollary 3.

Proof. Put $\widetilde{E}=\tilde{p}^{-1}(\infty)$. Let $\widetilde{H}_{k} \cap \widetilde{E}=a_{k}$. By Lemma $4 a_{1} \neq a_{2}$. Note that, since $\widetilde{H}_{k}$ is not a section, $\widetilde{H}_{k}$ does not meet $\widetilde{E}$ normally at $a_{k}$ (indeed, $\tilde{p}^{-1}(c) \cdot \widetilde{H}_{k} \geq 2$ for every $c \in \mathbf{C P}^{1}$, since $\widetilde{H}_{k}$ is not a section, and, in particular, $\widetilde{H}_{k} \cdot \widetilde{E} \geq 2$ ). Thus in order to obtain $\widehat{X}$ we must make blow-ups of $\tilde{X}$ at both the points $a_{1}$ and $a_{2}$, and at infinitely near points. Note then $\widetilde{\mathscr{D}} \backslash \widetilde{E}$ consists at least of two connected components, since $\widetilde{\mathscr{D}}$ is simply connected. Hence, if $g$ is the vertex of $G(\widehat{\mathscr{D}})$ that corresponds to the proper transform of $\widetilde{E}$, then the graph obtained by removing $g$ from the vertices of $G(\widehat{\mathscr{D}})$ and deleting the links at $g$ contains at least two components $C_{1}$ and $C_{2}$. Each of these components $C_{k}$ contains a vertex $f_{k}$ with the weight -1 (this vertex is the result of the last blow-up in this component). Remark that vertex $f_{k}$ is not a linear point. Otherwise we can contract the curve corresponding to $f_{k}$ and all points of selfintersection remain simple normal. The vertices $f_{1}$ and $f_{2}$ are not neighboring, since there is at least the vertex $g$ between them. The lemma is proved.

Corollary 3, Lemmas 4 and 5 immediately imply the following theorem.

TheOREM 5. Let $p: \mathbf{C}^{2} \rightarrow \mathbf{C}$ be a rational polynomial and $\bar{p}: \bar{X} \rightarrow$ $\mathbf{C}^{2}$ be the regular extension of $p$ to a compactification $\bar{X}$ of $\mathbf{C}^{2}$. Then, at most one horizontal component of the curve $\mathscr{D}=\bar{X}-\mathbf{C}^{2}$ is not a section.

EXAMPLE. Rational polynomials with a horizontal component different from a section do exist, but they are not simple. They were first found among polynomials with generic fiber isomorphic to $\mathbf{C}^{* *}=$ $\mathbf{C}-\{0,1\}$ (we shall refer to these polynomials as $\mathbf{C}^{* *}$ polynomials). An algebraic curve is a $\mathbf{C}^{*}$-curve if it is isomorphic to $\mathbf{C}^{*}$. 
We say that a $\mathbf{C}^{* *}$-polynomial is a Saito polynomial if its degenerate fibers contains at least one component different from a $\mathbf{C}^{*}$-curve. One can find the list of these polynomials up to a polynomial automorphism of $\mathbf{C}^{2}$ in [S]. There is also a wrong assertion in [S] that every $\mathbf{C}^{* *}$-polynomial is a Saito polynomial. Recently M. G. Zaidenberg [Z2] found all $\mathbf{C}^{* *}$-polynomials whose degenerate fibers consist of $C^{*}$-curves only. We shall call them Zaidenberg's polynomials. Every Zaidenberg's polynomial has two degenerate fibers. One of them (say $\left.\Gamma_{0}\right)$ is a $\mathbf{C}^{*}$-curve and another is the disjoint union of two $\mathbf{C}^{*}$-curves. Since $\Gamma_{0}$ is a twice-punctured Riemann surface, every Zaidenberg's polynomial has at most two horizontal components (in fact it has exactly two horizontal components, because every rational polynomial with one horizontal component is equivalent to a linear polynomial up to a polynomial automorphism of $\mathbf{C}^{2}$ [S]). On the other hand one of these horizontal components must be different from a section (otherwise the generic fiber must be a $\mathbf{C}^{*}$-curve as well). For the sake of completeness we shall present the simplest Zaidenberg polynomial and repeat some arguments from [Z2]. Let $\sigma(x, y)=x y+1$; then $p(x, y)=4\left(\sigma^{2}+y\right)(x \sigma+1)^{2}+1$ is a Zaidenberg polynomial.

Put $\Gamma_{c}=\{(x, y) \mid p(x, y)=c\}$. It is easy to check that $\Gamma_{1}$ consists of two disjoint $\mathbf{C}^{*}$-curves. In order to prove that the generic fiber of $p$ is $\mathbf{C}^{* *}$, consider $\rho(x, y)=x \sigma+1$. Then $\rho(x, y) \neq 0$ when $(x, y) \notin \Gamma_{1}$, and one can rewrite the equation for $\Gamma_{c}$ with $c \neq 1$ in the following form:

$$
c^{\prime} x^{2}-\rho^{2} x+\rho^{2}(\rho-1)=0,
$$

where $c^{\prime}=(c-1) / 4$. Consider the mapping $\left.\rho\right|_{\Gamma_{c}}: \Gamma_{c} \rightarrow \mathbf{C}^{*}$. If $c \neq 0,1$, then for every $s \in \mathbf{C}^{*}$ the inverse image $\left(\left.\rho\right|_{\Gamma_{c}}\right)^{-1}(s)$ consists of two points with $x=\left(1 / 2 c^{\prime}\right)\left(-s^{2} \pm s \sqrt{c s\left(s-s_{0}\right)}\right)$, where $s_{0}=1-1 / c$ is the only branch point in $\mathbf{C}^{*}$. Study of monodromy implies that there are two punctures on $\Gamma_{c}$ over $s=\infty$ and one puncture over $s=0$. It follows from the Riemann-Gurwith formula that $\Gamma_{c}$ is isomorphic to $\mathbf{C}^{* *}$. The same argument shows that $\left.\rho\right|_{\Gamma_{0}}: \Gamma_{0} \rightarrow \mathbf{C}^{*}$ is an unramified covering and, therefore, $\Gamma_{0}$ is a $\mathbf{C}^{*}$-curve. Every Zaidenberg polynomial is isotrivial, i.e., its generic fibers are isomorphic. More complicated examples of nonisotrivial rational polynomials with a $\mathbf{C}^{*}$-fiber (and, thus, with a horizontal component different from a section) are presented in $[\mathbf{K}]$.

It is a pleasure to thank V. Lin and M. Zaidenberg for stimulating discussions. 


\section{REFERENCES}

[AM] S. S. Abhyankar and T. T. Moh, Embeddings of the line in the plane, J. Reine Angew. Math., 276 (1975), 148-166.

[AS] S. S. Abhyankar and B. Singh, Embeddings of certain curves, Amer. J. Math., 100 (1978), 99-175.

[F] M. Furushima, Finite groups of polynomial automorphisms in the complex affine plane, I, Mem. Fac. Sci., Kyushu Univ., Ser. A, 36 (1982), 85-105.

[GH] Ph. Griffiths and J. Harris, Principles of Algebraic Geometry, John Wiley \& Sons, New York-Chichester-Brisbane-Toronto, 1978.

[K] Sh. Kaliman, Rational polynomials with a $\mathbf{C}^{*}$-fiber, Abstracts Amer. Math. Soc., 12 (1991), p. 44.

[LZ] V. Lin and M. Zaidenberg, An irreducible simply connected curve in $\mathbf{C}^{2}$ is equivalent to a quasihomogeneous curve, Soviet Math. Dokl., 28 (1983), 200204.

[L] S. Loyasiewicz, Triangulation of semianalytic sets, Ann. Scuola Norm. Sup. Pisa, Sc. Fis. Mat. Ser. 3, 18 (1964), 449-474.

[M] J. A. Morrow, Compactification of $\mathbf{C}^{2}$, Bull. Amer. Math. Soc., 78 (1972), 813-816.

[N] W. D. Neumann, Complex algebraic plane curves via their links at infinity, Invent. Math., 98 (1989), 445-489.

[R] C. P. Ramanujam, $A$ topological characterization of the affine plane as an algebraic variety, Ann. of Math., 94 (1971), 69-88.

[S] H. Saito, Fonctions entieres qui se réduisent à certains polynômes, II, Osaka J. Math., 14 (1977), 649-674.

[Sh] I. R. Shafarevich et al., Algebraic surfaces, Trudy Mat. Inst. Steklov., 75 (1965); English transl., Proc. Steklov Inst. Math., 75 (1965).

[Sp] E. H. Spanier, Algebraic Topology, Springer-Verlag, New York-HeidelbergBerlin, 1966.

[St] J. Stein, The total reducibility order of a polynomial in two variables, Tel-Aviv University, preprint, 1989.

[Z1] M. Zaidenberg, Isotrivial families on affine surfaces and characterization of the affine plane, Math. USSR Izvestiya, 30 (1988), 503-532.

[Z2] _ On Ramanujam surfaces, Soviet Math. Dokl., 314 (1990), 1303-1307 (in Russian).

Received December 27, 1990.

Wayne State University

DETROIT, MI 48202 



\section{PACIFIC JOURNAL OF MATHEMATICS EDITORS}

\author{
V. S. VARADARAJAN \\ (Managing Editor) \\ University of California \\ Los Angeles, CA 90024-1555 \\ Herbert Clemens \\ University of Utah \\ Salt Lake City, UT 84112 \\ F. Michael Christ \\ University of California \\ Los Angeles, CA 90024-1555 \\ THOMAS ENRIGHT \\ University of California, San Diego \\ La Jolla, CA 92093
}

\author{
Nicholas ERcolani \\ University of Arizona \\ Tucson, AZ 85721 \\ R. FINN \\ Stanford University \\ Stanford, CA 94305 \\ VAughan F. R. JONES \\ University of California \\ Berkeley, CA 94720 \\ C. C. Moore \\ University of California \\ Berkeley, CA 94720 \\ MARTIN ScharlemanN \\ University of California \\ Santa Barbara, CA 93106 \\ Harold Stark \\ University of California, San Diego \\ La Jolla, CA 92093
}

STEVEN KeRCKHOFF

Stanford University

Stanford, CA 94305

\begin{tabular}{|c|c|c|c|c|}
\hline \multicolumn{5}{|c|}{ ASSOCIATE EDITORS } \\
\hline R. Arens & $\begin{array}{ll}\text { E. F. BECKENBACH } & \text { B. H } \\
(1906-1982) & \end{array}$ & NeUmanN & $\begin{array}{c}\text { F. WoLF } \\
(1904-1989)\end{array}$ & K. Yoshida \\
\hline \multicolumn{5}{|c|}{ SUPPORTING INSTITUTIONS } \\
\hline \multicolumn{2}{|c|}{ UNIVERSITY OF ARIZONA } & \multicolumn{3}{|c|}{ UNIVERSITY OF OREGON } \\
\hline \multicolumn{2}{|c|}{ UNIVERSITY OF BRITISH COLUMBIA } & \multicolumn{3}{|c|}{ UNIVERSITY OF SOUTHERN CALIFORNIA } \\
\hline \multicolumn{2}{|c|}{ CALIFORNIA INSTITUTE OF TECHNOLOGY } & \multicolumn{3}{|c|}{ STANFORD UNIVERSITY } \\
\hline \multicolumn{2}{|c|}{ UNIVERSITY OF CALIFORNIA } & \multicolumn{3}{|c|}{ UNIVERSITY OF HAWAII } \\
\hline \multicolumn{2}{|c|}{ MONTANA STATE UNIVERSITY } & \multicolumn{3}{|c|}{ UNIVERSITY OF TOKYO } \\
\hline \multicolumn{2}{|c|}{ UNIVERSITY OF NEVADA, RENO } & \multicolumn{3}{|c|}{ UNIVERSITY OF UTAH } \\
\hline & \multicolumn{3}{|c|}{ WASHINGTON STATE UNIVERSITY } \\
\hline \multicolumn{2}{|c|}{ OREGON STATE UNIVERSITY } & \multicolumn{3}{|c|}{ UNIVERSITY OF WASHINGTON } \\
\hline
\end{tabular}

The Supporting Institutions listed above contribute to the cost of publication of this Journal, but they are not owners or publishers and have no responsibility for its content or policies.

Mathematical papers intended for publication in the Pacific Journal of Mathematics should be in typed
form or offset-reproduced (not dittoed), double spaced with large margins. Please do not use built up fractions
in the text of the manuscript. However, you may use them in the displayed equations. Underline Greek letters
in red, German in green, and script in blue. The first paragraph must be capable of being used separately as
a synopsis of the entire paper. In particular it should contain no bibliographic references. Please propose a
heading for the odd numbered pages of less than 35 characters. Manuscripts, in triplicate, may be sent to any
one of the editors. Please classify according to the 1991 Mathematics Subject Classification scheme which
can be found in the December index volumes of Mathematical Reviews. Supply name and address of author
to whom proofs should be sent. All other communications should be addressed to the managing editor, or
Elaine Barth, University of California, Los Angeles, California $90024-1555-05$.
There are page-charges associated with articles appearing in the Pacific Journal of Mathematics. These
charges are expected to be paid by the author's University, Government Agency or Company. If the author or
authors do not have access to such Institutional support these charges are waived. Single authors will receive
50 free reprints; joint authors will receive a total of 100 free reprints. Additional copies may be obtained at
cost in multiples of 50 .

The Pacific Journal of Mathematics (ISSN 0030-8730) is published monthly except for July and August. Regular subscription rate: $\$ 190.00$ a year (10 issues). Special rate: $\$ 95.00$ a year to individual members of supporting institutions.

Subscriptions, orders for numbers issued in the last three calendar years, and changes of address should be sent to Pacific Journal of Mathematics, P.O. Box 969, Carmel Valley, CA 93924, U.S.A. Old back numbers obtainable from Kraus Periodicals Co., Route 100, Millwood, NY 10546.

The Pacific Journal of Mathematics at P.O. Box 969, Carmel Valley, CA 93924 (ISSN 0030-8730) is published monthly except for July and August. Second-class postage paid at Carmel Valley, California 93924, and additional mailing offices. Postmaster: send address changes to Pacific Journal of Mathematics, P.O. Box 969, Carmel Valley, CA 93924.

PUBLISHED BY PACIFIC JOURNAL OF MATHEMATICS, A NON-PROFIT CORPORATION Copyright (C) 1992 by Pacific Journal of Mathematics 


\section{Pacific Journal of Mathematics}

\section{Vol. 154, No. $2 \quad$ June, 1992}

Manuel (Rodriguez) de León, J. A. Oubiña, P. R. Rodrigues and

Modesto R. Salgado, Almost $s$-tangent manifolds of higher order . . . . 201

Martin Engman, New spectral characterization theorems for $S^{2} \ldots \ldots \ldots 215$

Yuval Zvi Flicker, The adjoint representation $L$-function for GL $(n) \ldots \ldots 231$

Enrique Alberto Gonzalez-Velasco and Lee Kenneth Jones, On the range

of an unbounded partly atomic vector-valued measure . ............ 245

Takayuki Hibi, Face number inequalities for matroid complexes and

Cohen-Macaulay types of Stanley-Reisner rings of distributive

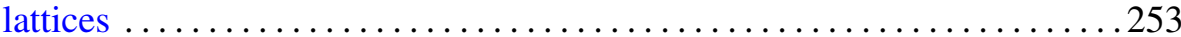

Hervé Jacquet and Stephen James Rallis, Kloosterman integrals for skew

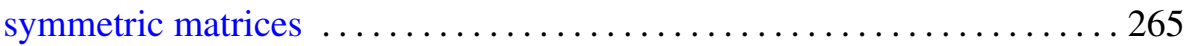

Shulim Kaliman, Two remarks on polynomials in two variables .........285

Kirk Lancaster, Qualitative behavior of solutions of elliptic free boundary problems ..........................................297

Feng Luo, Actions of finite groups on knot complements . ........... 317

James Joseph Madden and Charles Madison Stanton, One-dimensional

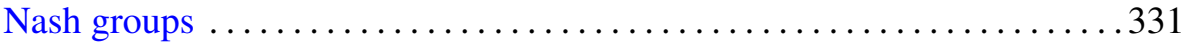

Christopher K. McCord, Estimating Nielsen numbers on

infrasolvmanifolds ......................................... 345

Gordan Savin, On the tensor product of theta representations of $\mathrm{GL}_{3}$ 369

Gerold Wagner, On means of distances on the surface of a sphere. II.

(Upper bounds) 